\title{
CONSTRUÇÃO DE INSTRUMENTO PARA ASSISTÊNCIA À SAÚDE DA MULHER NA ATENÇÃO PRIMÁRIA À SAÚDE
}

\author{
INSTRUMENT CONSTRUCTION FOR HEALTH CARE \\ WOMAN IN PRIMARY HEALTH CARE
}

\section{Rita de Cássia Rodrigues', Stella Crisanto Pontes', Mayhara Myrna Bezerril Freire de Lima Galvão', Fábia Barbosa de Andrade², Daísy Vieira de Araújo³.}

\begin{abstract}
RESUMO
O objetivo do presente estudo foi desenvolver um instrumento para assistência à saúde da mulher na Atenção Primária do município de Santa Cruz, Rio Grande do Norte. Trata-se de uma pesquisa exploratória e descritiva, com abordagem quantitativa. Participaram nove enfermeiros como avaliadores do estudo. Houve um consenso de mais de $50 \%$ entre os expertises. Calculou-se 0 coeficiente Alfa de Cronbach dos itens: identificação da paciente e informação sobre saúde-doença; exame físico; diagnóstico de enfermagem e intervenções da Ficha Clínica de Saúde da Mulher e obteve-se um resultado igual a 0,971 (97,1\%), 0,917 (91,7\%), 0,960 (96,1\%), 1,000 (100\%) e 1,000 $(100 \%)$, respectivamente, o que significa que os dados utilizados possuem validade interna, ou seja, o constructo é fidedigno. Os avaliadores participaram de forma assídua da elaboração do instrumento e, assim, forneceram uma ficha clínica com agrupamento de informações do estado de saúde-doença, atribuindo uma melhor qualificação à assistência prestada.
\end{abstract}

Descritores: Saúde da Mulher; Assistência de Enfermagem; Assistência Integral à Saúde da Mulher

\begin{abstract}
The aim of this study was to develop a tool for health care of women in primary care in the city of Santa Cruz, Rio Grande do Norte. This is an exploratory and descriptive research with a quantitative approach. Nine nurses participated as expertise of the study. There was a more than $50 \%$ consensus between expertise. Cronbach Alpha coefficient was calculated items: patient identification and health information and disease; physical exam; nursing diagnosis and interventions Sheet Health Clinic for Women and obtained a score of $0.971(97.1 \%), 0.917(91.7 \%), 0,960$ (96.1\%), 1,000 (100\%) and $1.000(100 \%)$, respectively, which means that the data used have internal validity, namely, the construct is trusted. The expertise assiduously participated in the drafting of the instrument and thus provided a clinical form with grouping of health information - disease, giving a better qualification to the assistance provided.
\end{abstract}

Descriptors: Women's Health; Nursing Care; Comprehensive Health Care woman
1 Graduada em Enfermagem pela Universidade Federal do Rio Grande do Norte (UFRN), Santa Cruz, RN, Brasil.

${ }^{2}$ Doutora em Ciências da Saúde pela Universidade Federal do Rio Grande do Norte (UFRN), Natal, RN, Brasil.

${ }^{3}$ Doutoranda em Ciências da Saúde pela Universidade Federal do Rio Grande do Norte (UFRN), Natal, RN, Brasil. 


\section{Introdução}

A figura feminina é de suma importância no processo saúde - doença, pois, na maioria dos casos, é responsável por manter a saúde da família. A população brasileira é constituída por $51,04 \%$ de mulheres ${ }^{1}$, sendo as principais usuárias do Sistema Único de Saúde (SUS), com maior frequência, em busca de atendimento para filhos, netos, marido, idosos e vizinhos e, muitas vezes, esquecendo-se do seu próprio cuidado, tornando-se vulnerável às doenças.

Atualmente, as mulheres têm ocupado o mercado de trabalho assumindo diversos papéis e múltiplas responsabilidades e, em virtude disso, para elas, tem restado pouco tempo para cuidar de si, uma vez que acabam dando prioridade às necessidades dos outros e colocando sua saúde em segundo plano².

Segundo o Sistema de Informações sobre mortalidade (SIM/DATASUS), no período de 2009 a 2013, em Santa Cruz/RN, foram registrados 425 óbitos de mulheres na faixa etária de 10 a 80 anos e mais, sendo as principais causas as doenças do aparelho circulatório, neoplasias (tumores), seguidas por doenças endócrinas, nutricionais e metabólicas ${ }^{3}$.

No período de janeiro de 2013 a setembro de 2015, no município de Santa Cruz, segundo o Sistema de Internações Hospitalares (SIH/ DATASUS), foram registrados 5.551 casos de internações relacionados à gravidez, parto e puerpério; seguido de 373 casos de doenças do aparelho digestório; 332 casos de doenças do aparelho geniturinário; 310 casos de internações por doenças do aparelho respiratório, em mulheres na faixa etária de 10 a 80 anos e mais ${ }^{4}$.

Nesse contexto, dados como esses despertam interesse em desenvolver um estudo centrado no atendimento à saúde da mulher, tendo em vista também a lacuna bibliográfica existente acerca de instrumentos que norteiem a ação do cuidado de enfermagem na saúde da mulher na atenção primária.

Desta forma, o desenvolvimento deste estudo se justifica pela necessidade de unificação de informações acerca do processo saúde-doença das mulheres atendidas na atenção primária, bem como dispor ao município de Santa Cruz/RN uma ficha clínica de saúde da mulher que contenha indicadores importantes para mensurar, avaliar e propor adequações aos programas municipais voltados a esta clientela, já que o município não apresenta histórico da utilização de um instrumento específico voltado para a mulher.

Assim, a ficha clínica proposta neste estudo contribuirá para uma melhora na qualidade da assistência de enfermagem prestada à mulher, de modo que se evite a duplicidade de informações nos prontuários. Igualmente, contribuirá para que a assistência se dê de forma holística e resolutiva, haja vista que possibilitará o desenvolvimento de um plano assistencial individual, além de fortalecer a interação enfermeiro-usuária do SUS.

Portanto, o objetivo do estudo foi desenvolver um instrumento voltado para a assistência à saúde da mulher na atenção primária do município de Santa Cruz, Rio Grande do Norte.

\section{Metodologia}

O presente estudo foi desenvolvido no município de Santa Cruz, localizado no estado do Rio Grande do Norte, a $111 \mathrm{~km}$ da capital, Natal. Trata-se de uma pesquisa exploratória e descritiva, com abordagem quantitativa.

O Sistema de Saúde do município presta serviços e ações em dois níveis, primário e secundário, e está organizado a partir da atenção primária à saúde, tendo-a como sua porta de acesso. Esta rede possui 6 unidades de saúde, com 12 equipes de Saúde da Família, sendo 10 na zona urbana e 2 na zona rural.

Os avaliadores foram escolhidos conforme os seguintes critérios: ser enfermeiro da Unidade Básica de Saúde do município de Santa Cruz e ter experiência nas áreas de saúde da mulher e atenção primária. Foram escolhidos nove enfermeiros de ambos os sexos para participar como avaliadores do estudo, com idade entre 24 e 33 anos e tempo de formação mínima de três anos.

O projeto foi submetido e aprovado pelo Comitê de Ética em Pesquisa da Faculdade de Ciências da Saúde do Trairi, da Universidade Federal do Rio Grande do Norte (UFRN), sob parecer de número 1.002.116, e atendeu aos requisitos e diretrizes éticas da pesquisa envolvendo seres humanos, recomendadas pela Comissão Nacional de Ética em Pesquisa (CONEP), expressas na Resolução nº 466/12 do Conselho Nacional de Saúde ${ }^{5}$.

Os avaliadores foram informados sobre os objetivos do estudo e a justificativa da pesquisa. Além disso, foram esclarecidos de que a participação era voluntária, havendo a liberdade para se retirar do estudo durante ou depois da finalização do processo de investigação, sem risco de qualquer penalização ou prejuízo de natureza pessoal ou 
profissional. Também, foi assegurado aos participantes o anonimato quando da publicação dos resultados, bem como 0 sigilo de informações consideradas confidenciais.

O presente estudo foi dividido em quatro etapas, com base na técnica Delphi6.

$\mathrm{Na} 1^{a}$ etapa, foi elaborado um instrumento modelo, uma ficha clínica de saúde da mulher, com o objetivo de coletar dados sobre as mulheres atendidas na atenção primária. Este instrumento teve como embasamento teórico a anamnese e o exame físico completos voltados para a atenção à saúde da mulher, levando em consideração as etapas do processo de enfermagem (coleta de dados, diagnósticos de enfermagem, intervenção, implementação e evolução).

Já a $2^{\mathrm{a}}$ etapa referiu-se à avaliação dos itens contidos no instrumento modelo. Os avaliadores poderiam acrescentar ou propor modificações aos itens do instrumento em questão.

Para tanto, foi entregue uma ficha de avaliação com os critérios de organização, objetividade, clareza e compreensão do conteúdo, e eles deveriam escolher uma alternativa dentre: 1 discordo totalmente; 2 discordo; 3 não concordo e nem discordo; 4 concordo e 5 concordo totalmente. Ao final da ficha, os avaliadores poderiam fazer comentários e sugestões. 0 instrumento modelo, juntamente com a ficha de avaliação, passou um período de três semanas a um mês com os avaliadores.

A $3^{a}$ etapa serviu para reformular o instrumento modelo. A partir da análise do instrumento modelo e da ficha de avaliação, adotando um nível de consenso de $50 \%$ das respostas dadas, o instrumento foi reformulado.

Por fim, a $4^{a}$ etapa associou-se à elaboração do instrumento final. Assim, os itens que obtiveram nível de consenso de $50 \%$ das respostas pelos avaliadores foram mantidos, finalizando, portanto, o processo de elaboração do instrumento final, que resultou na ficha clínica de saúde da mulher.

Após o encerramento de todas as etapas, a pesquisadora e aluna participante enviaram uma carta endereçada a cada avaliador agradecendo a participação no estudo, bem como o envio de um convite à Secretaria Municipal de Saúde e aos enfermeiros da APS de Santa Cruz para assistirem à defesa de trabalho de conclusão de curso da aluna.

Para a análise dos dados descritivos e Alfa de Cronbach, foi utilizado o pacote estatístico SPSS versão 20.0. O coeficiente Alfa de Cronbach é um índice utilizado para estimar a confiabilidade de uma escala, ou seja, avaliar a magnitude em que os itens de um instrumento estão correlacionados. Em outras palavras, pode-se afirmar que seu resultado é a média das correlações entre os itens que compõem um instrumento ${ }^{7}$.

O menor valor aceitável para esse coeficiente é de 0,70 (70\%). Valores abaixo desse significam que a consistência interna do instrumento utilizado é baixa?

\section{Resultados e Discussão}

Na análise realizada acerca da avaliação do instrumento pelos avaliadores, foi nítida a aceitação da ficha clínica de saúde da mulher. A tabela 1 apresenta a distribuição das frequências absoluta e relativa acerca dos atributos identificação da paciente e informações sobre saúde doença.

Tabela 1 - Distribuição das frequências absoluta e relativa dos atributos identificação da paciente e informações sobre saúde - doença da ficha clínica de saúde da mulher. Santa Cruz, Brasil, 2015.

\begin{tabular}{c|c|c|c|c|c}
\hline $\begin{array}{c}\text { IDENTIFICAÇÃo } \\
\text { DA PACIENTE }\end{array}$ & $\begin{array}{c}\text { Discordo } \\
\text { totalmente } \\
\mathbf{n}(\%)\end{array}$ & $\begin{array}{c}\text { Discordo } \\
\mathbf{n}(\%)\end{array}$ & $\begin{array}{c}\text { Não concordo e } \\
\text { Nem discordo } \\
\mathbf{n}(\%)\end{array}$ & $\begin{array}{c}\text { Concordo } \\
\mathbf{n}(\%)\end{array}$ & $\begin{array}{c}\text { Concordo } \\
\text { totalmente } \\
\mathbf{n}(\%)\end{array}$ \\
\hline Organização & 0 & 0 & 0 & $6(66,7)$ & $3(33,3)$ \\
\hline Objetividade & 0 & 0 & 0 & $6(66,7)$ & $3(33,3)$ \\
\hline Clareza & 0 & 0 & $1(11,1)$ & $5(55,6)$ & $3(33,3)$ \\
\hline Compreensão & 0 & 0 & $1(11,1)$ & $5(55,6)$ & $3(33,3)$ \\
\hline
\end{tabular}

Continua 


\begin{tabular}{c|c|c|c|c|c}
\hline $\begin{array}{c}\text { INFORMAÇÕES } \\
\text { SOBRE SAÚDE } \\
\text { DOENÇA }\end{array}$ & $\begin{array}{c}\text { Discordo } \\
\text { totalmente } \\
\mathbf{n ( \% )}\end{array}$ & $\begin{array}{c}\text { Discordo } \\
\mathbf{n ( \% )}\end{array}$ & $\begin{array}{c}\text { Não concordo e } \\
\text { Nem discordo } \\
\mathbf{n ( \% )}\end{array}$ & $\begin{array}{c}\text { Concordo } \\
\mathbf{n ( \% )}\end{array}$ & $\begin{array}{c}\text { Concordo } \\
\text { totalmente } \\
\mathbf{n ( \% )}\end{array}$ \\
\hline Organização & 0 & 0 & 0 & $6(66,7 \%)$ & $3(33,3 \%)$ \\
\hline Objetividade & 0 & 0 & 0 & $6(66,7 \%)$ & $3(33,3 \%)$ \\
\hline Clareza & 0 & $1(11,1 \%)$ & 0 & $5(55,6 \%)$ & $3(33,3 \%)$ \\
\hline Compreensão & 0 & $1(11,1 \%)$ & 0 & $5(55,6 \%)$ & $3(33,3 \%)$ \\
\hline
\end{tabular}

O instrumento Ficha Clínica de Saúde da Mulher proposto neste estudo inicia-se com a identificação da paciente, constando de nome, data de nascimento, telefone e, incluindo, dados que podem proporcionar fatores de risco relacionados ao processo saúde-doença da figura feminina, tais como: idade; raça; nacionalidade; naturalidade; estado civil; número de filhos; nível de escolaridade e ocupação.

Nesse contexto, a coleta de dados é primordial para o processo de enfermagem, cujo objetivo é constatar dados pertinentes ao processo saúde-doença do indivíduo atendido, bem como analisar o ambiente e a comunidade onde ele está inserido.

Após a identificação, seguiu-se a etapa de informações sobre saúde-doença, com a busca por dados necessários para caracterizar o indivíduo, permitindo sua identificação; bem como informações sobre saúde-doença, consentindo ao profissional de saúde o conhecimento dos meios e fatores que venham a influenciar o bem-estar da paciente atendida na atenção primária do município de Santa Cruz.

Formada por perguntas simples e claras, com o objetivo de facilitar a compreensão tanto do indivíduo questionado quanto do profissional enfermeiro, esta etapa constituiu-se de perguntas do tipo: quantidade de gestações; vida sexual ativa; uso de preservativos e característica do ciclo menstrual, permitindo que, durante a consulta, o enfermeiro realize orientações a respeito do planejamento familiar, bem como orientações sobre sexualidade e métodos de prevenção do câncer do colo do útero e de mama.

Calculando-se o coeficiente Alfa de Cronbach dos itens da identificação da paciente e as informações sobre saúde-doença, obteve-se um resultado igual a 0,971 (97,1\%) e 0,917 (91,7\%), respectivamente, o que significa que os dados utilizados possuem significância interna, ou seja, parte do constructo é fidedigno.

A tabela 2 apresenta os dados da distribuição das frequências absoluta e relativa do atributo exame físico, que é subdividido em medidas antropométricas; avaliação neurológica; cabeça e pescoço; sistema cardiocirculatório; sistema respiratório; exame das mamas; sistema digestório e avaliação genital.

Tabela 2 - Distribuição das frequências absoluta e relativa do atributo exame físico da ficha clínica de saúde da mulher. Santa Cruz, Brasil, 2015.

\begin{tabular}{c|c|c|c|c|c}
\hline MEDIDAS & $\begin{array}{c}\text { Discordo } \\
\text { totalmente } \\
\mathbf{n ( \% )}\end{array}$ & $\begin{array}{c}\text { Discordo } \\
\mathbf{n ( \% )}\end{array}$ & $\begin{array}{c}\text { Não concordo e } \\
\text { Nem discordo } \\
\mathbf{n ( \% )}\end{array}$ & $\begin{array}{c}\text { Concordo } \\
\mathbf{n ( \% )}\end{array}$ & $\begin{array}{c}\text { Concordo } \\
\text { totalmente } \\
\mathbf{n}(\%)\end{array}$ \\
\hline Organização & 0 & 0 & 0 & $6(66,7)$ & $3(33,3 \%)$ \\
\hline Objetividade & 0 & 0 & 0 & $6(66,7)$ & $3(33,3 \%)$ \\
\hline Clareza & 0 & 0 & $7(11,1 \%)$ & $5(55,6 \%)$ & $3(33,3 \%)$ \\
\hline Compreensão & 0 & 0 & $1(11,1 \%)$ & $5(55,6 \%)$ & $3(33,3 \%)$ \\
\hline AVALIAÇÃo & $\begin{array}{c}\text { Discordo } \\
\text { totalmente } \\
\mathbf{n ( \% )}\end{array}$ & $\begin{array}{c}\text { Discordo } \\
\mathbf{n ( \% )}\end{array}$ & $\begin{array}{c}\text { Não concordo e } \\
\text { Nem discordo } \\
\mathbf{n ( \% )}\end{array}$ & $\begin{array}{c}\text { Concordo } \\
\mathbf{n ( \% )}\end{array}$ & $\begin{array}{c}\text { Concordo } \\
\text { totalmente } \\
\mathbf{n}(\%)\end{array}$ \\
\hline Organização & 0 & 0 & $4(44,4 \%)$ & $3(33,3 \%)$ & $2(22,2 \%)$ \\
\hline Objetividade & 0 & 0 & $4(44,4 \%)$ & $3(33,3 \%)$ & $2(22,2 \%)$ \\
\hline Clareza & 0 & 0 & $4(44,4 \%)$ & $2(22,2 \%)$ & $3(33,3 \%)$ \\
\hline Compreensão & 0 & 0 & $4(44,4 \%)$ & $2(22,2 \%)$ & $3(33,3 \%)$ \\
\hline
\end{tabular}




\begin{tabular}{|c|c|c|c|c|c|}
\hline CABEÇA E PESCOÇO & $\begin{array}{c}\text { Discordo } \\
\text { totalmente } \\
\mathbf{n}(\%) \\
\end{array}$ & $\begin{array}{l}\text { Discordo } \\
\mathbf{n}(\%)\end{array}$ & $\begin{array}{c}\text { Não concordo e } \\
\text { Nem discordo } \\
n(\%)\end{array}$ & $\begin{array}{l}\text { Concordo } \\
n(\%)\end{array}$ & $\begin{array}{c}\text { Concordo } \\
\text { totalmente } \\
\mathbf{n}(\%) \\
\end{array}$ \\
\hline Organização & 0 & 0 & $3(33,3 \%)$ & $5(55,6 \%)$ & $1(11,1 \%)$ \\
\hline Objetividade & 0 & 0 & $3(33,3 \%)$ & $5(55,6 \%)$ & $1(11,1 \%)$ \\
\hline Clareza & 0 & 0 & $3(33,3 \%)$ & $4(44,4 \%)$ & $2(22,2 \%)$ \\
\hline Compreensão & 0 & 0 & $3(33,3 \%)$ & $4(44,4 \%)$ & $2(22,2 \%)$ \\
\hline $\begin{array}{c}\text { SISTEMA } \\
\text { CARDIOCIRCULATÓRIO }\end{array}$ & $\begin{array}{c}\text { Discordo } \\
\text { totalmente } \\
\mathbf{n}(\%) \\
\end{array}$ & $\begin{array}{l}\text { Discordo } \\
\mathbf{n}(\%)\end{array}$ & $\begin{array}{c}\text { Não concordo e } \\
\text { Nem discordo } \\
n(\%)\end{array}$ & $\begin{array}{l}\text { Concordo } \\
\text { n(\%) }\end{array}$ & $\begin{array}{c}\text { Concordo } \\
\text { totalmente } \\
\mathrm{n}(\%) \\
\end{array}$ \\
\hline Organização & 0 & 0 & $3(33,3 \%)$ & $4(44,4 \%)$ & $2(22,2 \%)$ \\
\hline Objetividade & 0 & 0 & $3(33,3 \%)$ & $4(44,4 \%)$ & $2(22,2 \%)$ \\
\hline Clareza & 0 & 0 & $3(33,3 \%)$ & $3(33,3 \%)$ & $3(33,3 \%)$ \\
\hline Compreensão & 0 & 0 & $3(33,3 \%)$ & $3(33,3 \%)$ & $3(33,3 \%)$ \\
\hline $\begin{array}{c}\text { SISTEMA } \\
\text { RESPIRATÓRIO }\end{array}$ & $\begin{array}{c}\text { Discordo } \\
\text { totalmente } \\
\mathbf{n}(\%) \\
\end{array}$ & $\begin{array}{l}\text { Discordo } \\
\mathbf{n}(\%)\end{array}$ & $\begin{array}{c}\text { Não concordo e } \\
\text { Nem discordo } \\
\text { n(\%) }\end{array}$ & $\begin{array}{l}\text { Concordo } \\
\text { n(\%) }\end{array}$ & $\begin{array}{c}\text { Concordo } \\
\text { totalmente } \\
\text { n(\%) }\end{array}$ \\
\hline Organização & 0 & 0 & $2(22,2 \%)$ & $5(55,6 \%)$ & $2(22,2 \%)$ \\
\hline Objetividade & 0 & 0 & $2(22,2 \%)$ & $4(44,4 \%)$ & $3(33.3 \%)$ \\
\hline Clareza & 0 & 0 & $2(22,2 \%)$ & $4(44,4 \%)$ & $3(33.3 \%)$ \\
\hline Compreensão & 0 & 0 & $2(22,2 \%)$ & $4(44,4 \%)$ & $3(33.3 \%)$ \\
\hline EXAME DAS MAMAS & $\begin{array}{c}\text { Discordo } \\
\text { totalmente } \\
n(\%)\end{array}$ & $\begin{array}{l}\text { Discordo } \\
n(\%)\end{array}$ & $\begin{array}{c}\text { Não concordo e } \\
\text { Nem discordo } \\
n(\%)\end{array}$ & $\begin{array}{l}\text { Concordo } \\
\text { n(\%) }\end{array}$ & $\begin{array}{c}\text { Concordo } \\
\text { totalmente } \\
\text { n(\%) }\end{array}$ \\
\hline Organização & 0 & 0 & 0 & $2(22,2 \%)$ & $7(77,8 \%)$ \\
\hline Objetividade & 0 & 0 & 0 & $2(22,2 \%)$ & $7(77,8 \%)$ \\
\hline Clareza & 0 & 0 & 0 & $2(22,2 \%)$ & $7(77,8 \%)$ \\
\hline Compreensão & 0 & 0 & 0 & $2(22,2 \%)$ & $7(77,8 \%)$ \\
\hline SISTEMA DIGESTÓRIO & $\begin{array}{c}\text { Discordo } \\
\text { totalmente } \\
n(\%) \\
\end{array}$ & $\begin{array}{l}\text { Discordo } \\
\mathbf{n}(\%)\end{array}$ & $\begin{array}{c}\text { Não concordo e } \\
\text { Nem discordo } \\
\text { n(\%) }\end{array}$ & $\begin{array}{l}\text { Concordo } \\
n(\%)\end{array}$ & $\begin{array}{c}\text { Concordo } \\
\text { totalmente } \\
\mathbf{n}(\%) \\
\end{array}$ \\
\hline Organização & 0 & 0 & $3(33,3 \%)$ & $3(33,3 \%)$ & $3(33,3 \%)$ \\
\hline Objetividade & 0 & 0 & $3(33,3 \%)$ & $4(44,4 \%)$ & $2(22,2 \%)$ \\
\hline Clareza & 0 & 0 & $3(33,3 \%)$ & $3(33,3 \%)$ & $3(33,3 \%)$ \\
\hline Compreensão & 0 & 0 & $3(33,3 \%)$ & $3(33,3 \%)$ & $3(33,3 \%)$ \\
\hline AVALIAÇÃO GENITAL & $\begin{array}{c}\text { Discordo } \\
\text { totalmente } \\
\mathbf{n}(\%)\end{array}$ & $\begin{array}{l}\text { Discordo } \\
\mathbf{n}(\%)\end{array}$ & $\begin{array}{c}\text { Não concordo e } \\
\text { Nem discordo } \\
n(\%)\end{array}$ & $\begin{array}{l}\text { Concordo } \\
\text { n(\%) }\end{array}$ & $\begin{array}{c}\text { Concordo } \\
\text { totalmente } \\
\mathrm{n}(\%)\end{array}$ \\
\hline Organização & 0 & 0 & 0 & $3(33,3 \%)$ & $6(66,7 \%)$ \\
\hline Objetividade & 0 & 0 & 0 & $2(22,2 \%)$ & $7(77,8 \%)$ \\
\hline Clareza & 0 & 0 & 0 & $3(33,3 \%)$ & $6(66,7 \%)$ \\
\hline Compreensão & 0 & 0 & 0 & $2(22,2 \%)$ & $7(77,8 \%)$ \\
\hline
\end{tabular}


O exame físico é desenvolvido no sentido cefalocaudal, iniciado com as medidas antropométricas, peso e altura, que permitem anotar o índice de massa corpórea e corroboram para a investigação do estado nutricional da paciente; pressão arterial, além de possibilitar anotação dos níveis pressóricos e, desta forma, verificar a possibilidade da paciente desenvolver doenças crônicas.

Em seguida, realiza-se a avaliação neurológica, com a avaliação do nível de consciência e a coordenação dos movimentos. Para isso, o enfermeiro deve observar as expressões verbais e os movimentos da paciente.

Quanto ao exame da cabeça e pescoço, devem ser avaliados o couro cabeludo, a acuidade visual, acuidade auditiva, as amígdalas, a tireoide, linfonodos e alterações da pele. No desenvolver do exame, o enfermeiro deve utilizar os métodos propedêuticos, inspeção e palpação, bem como observar sinais e sintomas que possam ser característicos de alguma enfermidade, visando ofertar uma assistência de qualidade ${ }^{8}$.

No tocante ao exame do sistema cardiocirculatório, é avaliado o pulso, que pode ser rítmico, arrítmico, cheio ou filiforme; a perfusão periférica; ausculta cardíaca, bem como a presença ou ausência de sopros. Devido a essa avaliação, o enfermeiro pode elaborar planos de cuidados para a mulher, a partir da verificação de possíveis cardiopatias, além de fornecer meios de tratamentos que venham a facilitar o convívio da paciente. Cabe lembrar que o indivíduo não deve ser fragmentando em partes, mas sim visto de forma holística.

No que concerne ao exame do sistema respiratório, sabe-se que é responsável pela captação de oxigênio do ambiente e transporte para os pulmões, troca de dióxido de carbono nos alvéolos e da devolução para 0 ambiente ${ }^{8}$. Na ficha clínica, é avaliada essa função através do estado respiratório: presença, diminuição ou ausência de murmúrio vesicular e presença ou ausência de ruídos adventícios. Esse exame fornece informações a respeito do padrão respiratório da paciente, como sua necessidade de suporte ventilatório, caso seja identificado.

$\mathrm{Na}$ avaliação das mamas, é realizada a inspeção e palpação, com intuito de avaliar a forma, tamanho, tipos de mamilos, presença ou ausência de secreção mamilar e nódulos, e contribuir para avaliar a indicação dos procedimentos de diagnóstico inicial e o rastreamento precoce do câncer de mama9.

Na fase de avaliação do sistema digestório, é observado o formato do abdômen, a presença de dor à palpação, massa palpável, ausculta abdominal e a presença ou ausência de eliminações intestinais. Para a realização do exame digestório, são utilizadas as técnicas propedêuticas da seguinte forma: inspeção, ausculta, percussão e palpação, permitindo uma melhor exatidão dos resultados obtidos com o exame ${ }^{8}$.

A avaliação genital é de suma importância para prevenção e tratamento de doenças sexualmente transmissíveis, bem como a prevenção do câncer do colo do útero e infecção do trato urinário. Para tanto, nessa fase, são avaliados os grandes e pequenos lábios, clitóris, meato uretral, introito vaginal, períneo, ânus, colo do útero e mucosa, com o auxílio do exame preventivo.

Nos países em desenvolvimento, o câncer de colo uterino é tido como uma das principais causas de morte. Está atrelado a diversos fatores que contribuem para esse fato, como ausência de educação em saúde, responsável pela detecção precoce; falta de adesão das mulheres a programas de prevenção; a elevada taxa de infecção pelo Papiloma Vírus Humano (HPV) e as diferenças culturais ${ }^{10}$.

Entende-se que a estratégia primária para prevenção de doenças dos genitais é o uso do preservativo e a secundária, o diagnóstico e tratamento ${ }^{11}$.

Calculando-se o coeficiente Alfa de Cronbach do atributo exame físico da Ficha Clínica de Saúde da Mulher, obteve-se um resultado igual a $0,960(96,1 \%)$, o que significa que os dados utilizados possuem validade interna.

$\mathrm{Na}$ tabela 3 verifica-se a distribuição das frequências absoluta e relativa dos atributos diagnósticos de enfermagem e intervenções, assim fornecendo dados de aprovação para esses quesitos de acordo com as respostas dos expertises. 
Tabela 3 - Distribuição das frequências absoluta e relativa dos atributos diagnósticos de enfermagem e intervenções da ficha clínica de saúde da mulher. Santa Cruz, Brasil, 2015

\begin{tabular}{|c|c|c|c|c|c|}
\hline $\begin{array}{l}\text { DIAGNÓSTICOS DE } \\
\text { ENFERMAGEM }\end{array}$ & $\begin{array}{c}\text { Discordo } \\
\text { totalmente } \\
\text { n(\%) }\end{array}$ & $\begin{array}{l}\text { Discordo } \\
\mathbf{n}(\%)\end{array}$ & $\begin{array}{c}\text { Não concordo e } \\
\text { Nem discordo } \\
\text { n(\%) }\end{array}$ & $\begin{array}{l}\text { Concordo } \\
\text { n(\%) }\end{array}$ & $\begin{array}{c}\text { Concordo } \\
\text { totalmente } \\
\text { n(\%) }\end{array}$ \\
\hline Organização & 0 & 0 & 0 & $3(33,3 \%)$ & $6(66,7 \%)$ \\
\hline Objetividade & 0 & 0 & 0 & $3(33,3 \%)$ & $6(66,7 \%)$ \\
\hline Clareza & 0 & 0 & 0 & $3(33,3 \%)$ & $6(66,7 \%)$ \\
\hline Compreensão & 0 & 0 & 0 & $3(33,3 \%)$ & $6(66,7 \%)$ \\
\hline INTERVENÇÕES & $\begin{array}{c}\text { Discordo } \\
\text { totalmente } \\
\text { n(\%) } \\
\end{array}$ & $\begin{array}{l}\text { Discordo } \\
\mathbf{n}(\%)\end{array}$ & $\begin{array}{c}\text { Não concordo e } \\
\text { Nem discordo } \\
\text { n(\%) }\end{array}$ & $\begin{array}{l}\text { Concordo } \\
\text { n(\%) }\end{array}$ & $\begin{array}{c}\text { Concordo } \\
\text { totalmente } \\
\text { n(\%) }\end{array}$ \\
\hline Organização & 0 & 0 & 0 & $5(55,6 \%)$ & $4(44,4 \%)$ \\
\hline Objetividade & 0 & 0 & 0 & $5(55,6 \%)$ & $4(44,4 \%)$ \\
\hline Clareza & 0 & 0 & 0 & $5(55,6 \%)$ & $4(44,4 \%)$ \\
\hline Compreensão & 0 & 0 & 0 & $5(55,6 \%)$ & $4(44,4 \%)$ \\
\hline
\end{tabular}

Após o exame físico completo, a ficha segue com os diagnósticos de enfermagem e intervenções, que são essenciais para uma assistência de qualidade. Pois, é a partir do conhecimento dos problemas e agravos à saúde que são traçados meios que permitem o cuidado da paciente. Os diagnósticos de enfermagem devem ser anotados seguindo o modelo da NANDA ${ }^{12}$, e suas intervenções devem ser elaboradas no plano de cuidado, relacionadas a cada tipo de diagnóstico, com o intuito de coletar dados inerentes à paciente e à família e possibilitar uma visão biopsicossocial da mulher, para obter uma sistematização da assistência ampla e resolutiva.

Calculando-se o coeficiente Alfa de Cronbach dos atributos diagnóstico de enfermagem e intervenções da Ficha Clínica de Saúde da Mulher, obteve-se um resultado igual a 1,000 (100\%), o que significa que os dados utilizados possuem significância interna.

A sistematização da assistência de enfermagem deve ser bem embasada, e a coleta de dados desenvolvida de forma sistemática, respeitando a subjetividade do indivíduo. O papel do profissional de enfermagem é fundamental para o desenvolvimento de uma assistência de qualidade, adotando uma metodologia prática, clara e que seja condizente com a realidade do ambiente onde a paciente esteja inserida ${ }^{13}$.

A ficha clínica foi elaborada com o espaço destinado à anotação dos diagnósticos de enfermagem e intervenções, assim, possibilitando ao profissional que a utilize na sua assistência, analisar seu paciente no contexto do processo saúde-doença, respeitando a subjetividade e fornecendo diagnósticos e intervenções conforme a sua realidade.

A elaboração de um instrumento com obtenção de dados em saúde-doença possibilita documentar informações de forma clara e objetiva, fornecer identificação de diagnósticos de enfermagem, bem como as intervenções para uma assistência de qualidade ${ }^{14}$.

Os serviços de saúde eram mantidos na fragmentação do paciente. Com a instituição do Sistema Único de Saúde, o paciente passou a ser visto de maneira integral, visando à organização e articulação entre os serviços, corroborando para que a assistência seja articulada em seus diversos níveis de complexidade, bem como a articulação entre serviços privados e públicos, admitindo a resolução de problemas que venham a afetar a qualidade de vida individual ou coletiva, além de danos sofridos pela população em seu contexto de saúde ${ }^{15}$.

Os serviços de saúde da atenção básica se caracterizam pela união de ações voltadas para 0 individual e 0 coletivo, abrangendo a promoção, proteção e prevenção de agravos associados ao processo saúde-doença, com 0 intuito de estabelecer uma atenção integral ${ }^{16}$.

Nessa perspectiva, a ficha clínica foi elaborada objetivando estabelecer uma atenção integral à saúde da mulher, prestar uma assistência humanizada, holística, que considere o contexto biopsicossocial, atendendo aos princípios do SUS. Logo, possibilitar a ação de diminuir ou amenizar os problemas de saúde associados ao gênero feminino, torna-se cada vez mais necessário o preparo dos profissionais da saúde para enfrentar situações diversas que causem danos/agravos à saúde da mulher. 
Em busca de estudos desenvolvidos em relação a instrumentos voltados à atenção à saúde da mulher, foi possível encontrar um relato sobre a construção de instrumento de consulta à saúde da mulher ${ }^{17}$, o qual abordava a avaliação das mamas, abdominal e órgãos genitais; outro voltado para a consulta de enfermagem em sexualidade ${ }^{18}$; um para consulta de enfermagem à puérpera no âmbito da atenção básica 19 e, por fim, um sobre a construção de um instrumento de coleta de dados de pacientes internadas para cirurgias ginecológicas ${ }^{14}$.

Logo, não foi encontrado um instrumento que permitisse a assistência plena da saúde da mulher, de forma integral, sem fragmentação, o que faz com que o presente estudo seja visto como relevante e pertinente para a assistência de forma sistemática à saúde integral da mulher.

\section{Considerações Finais}

A ficha clínica de atenção à saúde da mulher mostrou-se adequada para a coleta de informações sobre o processo saúde-doença, uma vez que foi aprovada pelos avaliadores com nível de consenso superior a 50\%. Reconhece-se que a ficha foi bem elaborada e sua aplicabilidade foi considerada pertinente para a assistência integral prestada às pacientes atendidas na atenção primária à saúde.

Cabe destacar que este estudo apresentou uma limitação em relação à validação do construto e que, por isso, sugere-se a realização de novas pesquisas objetivando a validação do mesmo.

\section{Referências}

1. Instituto Brasileiro de Geografia e Estatística. Censo 2010: População do Brasil. 2010. [Acesso em 2015 Jan 28]. Disponível em: http://ibge.gov.brl

2. Smeltzer, Suzanne C.et al. Bunner \& Suddarth, Tratado de enfermagem médico - cirúrgico. $12^{\circ}$ ed. Rio de Janeiro(RJ): Guanabara Koogan; 2014.

3. Ministério da Saúde (BR). DATASUS - Departamento de Informática do SUS. Sistema de Informações sobre Mortalidade - SIM. [online] Brasília. . [Acesso em 2015 Nov 17]; Disponível em: http:/ttabnet.datasus.gov.br/cgi/tabcgi.exe?sim/cnv/obt10rn.def

4. Ministério da Saúde (BR). DATASUS - Departamento de Informática do SUS. Sistema de Informações Hospitalares do SUS (SIH/SUS) [Online] Brasília [Acesso em 2015 Nov 17]; Disponível em: http://tabnet.datasus.gov.br/cgi/tabcgi. exe?\%20sih/cnv/nirn.def

5. Ministério da Saúde (BR). Resolução n 466, de 12 de dezembro de 2012. [Acesso em 2015 Fev 15]. Disponível em: conselho.saude.gov.br/resolucoes/2012/Reso466.pdf.

6. Faro, A. C.M. e. Técnica Delphi na validação das intervenções de enfermagem. Rev.Esc.Enf.USP. 1997 [acesso em 2015 Fev 05]; 31(1); 259-73. Disponível em: http://www.ee.usp.br/reeusp/upload/pdf/415.pdf.

7. Oviedo, H. C.; arias, A. C. Aproximación al uso del coeficiente alfa de Cronbach. Revista Colombiana de Psiquiatria, 34(4). 2005. 8. Barros ALBL \& cols. Anamnese e exame físico: avaliação diagnóstica de enfermagem no adulto. 2. ed. Porto Alegre(RS): Artmed; 2010.

9. Brasil. Ministério da Saúde. Portaria no 779/08 de 31 de dezembro de 2008. Define o Sistema de Informação do Controle do Câncer de Mama (SISMAMA) como sistema oficial para o fornecimento dos dados informatizados dos procedimentos relacionados ao rastreamento e a confirmação diagnóstica do câncer de mama. Diário Oficial da União 2009; 2 jan. [acesso em 2015 Jan 13]. Disponível em http://www.normasbrasil.com.br/norma/portaria-779-2008_209559.html.

10. Diz MDPE, Medeiros RB de. Câncer de colo uterino - fatores de risco, prevenção, diagnóstico e tratamento. Rev Med (São Paulo). 2009 jan.-mar. [acesso em 2015 out 10] 88(1):7-15. Disponível em http://www.revistas.usp.br/revistadc/ article/viewFile/42183/45856.

11. Ministério da Saúde (BR). Secretaria de Vigilância em Saúde. Programa Nacional de DST e Aids. Manual de Controle das Doenças Sexualmente Transmissíveis/DST. Brasília: Ministério da Saúde. 2005. [acesso em 2015 out 10] http://www. aids.gov.br/sites/default/files/manual_dst_tratamento.pdf

12. Herdman, T. Heather (Org). Diagnósticos de enfermagem da NANDA International: definições e classificação, 20122014. Porto Alegre (RS): Artmed; 2013 
13. Bittar DB, Pereira LV, Lemos RCA. Sistematização da assistência de enfermagem ao paciente critico: Proposta de instrumento de coleta de dados. Texto Contexto Enferm. 2006[acesso em 2015 Out 10]; 15(4):617-28. Disponível em: http://www.scielo.br/pdf/tce/v15n4/v15n4a10

14. Soares LH, Pinelli FGS, Abrão ACFV. Construção de um instrumento de coleta de dados de enfermagem em ginecologia. Acta Paul Enferm. 2005 [Acesso em 2015 Out 10]; 18(2):156-64. Disponível em: http://www.scielo.br/pdf/ ape/v18n2/a07v18n2.pdf

15. Reis $\mathrm{CB}$, Andrade SMO. Representações sociais das enfermeiras sobre a integralidade na assistência à saúde da mulher na rede básica. Ciência \& Saúde coletiva. 2008 [acesso em 2015 Mar 02]; 13(1): 61-70. Disponível em http://www. scielo.br/pdf/csc/v13n1/10.pdf

16. Brasil, Ministério da Saúde. Portaria $n^{0}$ 2.488, de 21 de outubro de 2011. Aprova a Política Nacional de Atenção Básica, estabelecendo a revisão de diretrizes e normas para a organização da Atenção Básica, para a Estratégia Saúde da Família (ESF) e o Programa de Agentes Comunitários de Saúde (PACS). [Acesso em 2015 Fev 10]. Disponível em:http://bvsms.saude.gov.br/bvs/saudelegis/gm/2011/prt2488_21_10_2011.html

17. Oliveira FLB de, Araújo DV de, Meneses RMV. Relato sobre a construção de instrumento de consulta à mulher. Rev enferm UFPE on line, Recife, 8(6):1807-13, jun., 2014. [Acesso em 2014 Dez 20]. Disponível em: http://www.revista.ufpe. br/revistaenfermagem/index.php/revista/article/viewFile/4851/pdf_5381

18. Garcia ORZ, Lisboa LCS. Consulta de Enfermagem em sexualidade: Um instrumento para Assistência à saúde da mulher, em nível de atenção primária. Texto Contexto Enferm, Florianópolis, 2012 jul-set; 21(3):708-16. [acesso em 2015 jan 20]. Disponível em: http://www.scielo.br/scielo.php?script=sci_arttext\&pid=S0104-07072012000300028

19. Mazzo MHSN, Brito RS de. Validação de instrumento para consulta de enfermagem à puérpera no âmbito da atenção básica. Rev enferm UFPE on line. Recife, jul., 2013 [acesso em 2015 Jan 20]. 7(7):4809-13, Disponível em: http://www. revista.ufpe.br/revistaenfermagem/index.php/revista.

\section{Rita de Cássia Rodrigues}

Endereço para correspondência - Rua: João Bionor Bezerra, $n^{\circ} 310$, Bairro: Centro, CEP: 59200-000, Santa Cruz, Rio Grande do Norte, Brasil.

E-mail: cassiarodrigues_@live.com

Lattes: http://lattes.cnpq.br/6487475771523411

Stella Crisanto Pontes - stellafacisa@gmail.com

Mayhara Myrna Bezerril Freire de Lima Galvão - mayharamyrna@hotmail.com

Fábia Barbosa de Andrade - fabiabarbosabr@yahoo.com.br

Daísy Viera de Araújo - mestredaisy@yahoo.com.br

\section{Enviado em 30 de março de 2016. Aceito em 10 de junho de 2016.}


APÊNDICE A - FICHA CLÍNICA DE SAÚDE DA MULHER

UNIVERSIDADE FEDERAL DO RIO GRANDE DO NORTE - UFRN

FACULDADE DE CIÊNCIAS DA SAÚDE DO TRAIRI - FACISA

CURSO DE GRADUAÇÃO EM ENFERMAGEM

ASSISTÊNCIA À SAÚDE DA MULHER

NA ATENÇÃO PRIMÁRIA

Ficha Clínica de Saúde da Mulher

I. Identificação da paciente

Data da Consulta:

1

Nome:

Data de nascimento:

Idade:

Sexo: FEMININO

Raça:

Nacionalidade:

Naturalidade:

Estado civil:

Número de filhos:

Telefone:

Ocupação:

Nível de escolaridade:

II. Informações sobre saúde - doença

Queixa principal:

Corrimento vaginal: $\square \operatorname{Sim} \square$ Não Aspecto:__ Odor: $\square$ Sim $\square$ Não

Eliminações vesicais: $\square$ Normal $\square$ Diminuída $\square$ Dor $\square$ Ardor $\square$ prurido

Eliminação intestinal: $\square$ Presente $\square$ Ausente há ___ dias

Sono e Repouso: $\square$ Satisfatório $\square$ Insatisfatório Uso de medicação:

Gesta:_ Para:_ $\square$ Cesário $\square$ vaginal AB:

Idade da Menarca:

DUM:

Características do ciclo: $\square$ Regular $\square$ Irregular Presença de coágulos: $\square$ Sim $\square$ Não

Duração do sangramento:___ Presença de dismenorréia: $\square$ Sim $\square$ Não

Dispareunia: $\square \operatorname{Sim} \square$ Não Vida sexual ativa: $\square \operatorname{Sim} \square$ Não

Parceiro fixo: $\square \operatorname{Sim} \square$ Não Faz uso de preservativo: $\square \operatorname{Sim} \square$ Não

Faz uso de anticoncepcional oral ou injetável: $\square \operatorname{Sim} \square$ Não Qual:

Tempo:_ Já fez preventivo: $\square \operatorname{Sim} \square$ Não

Último preventivo:______ Tabagismo: $\square \operatorname{Sim} \square$ _ $\square$ _ _ Exo Ex-fumante: $\square \operatorname{Sim} \square$ Não

Período:_ Etilismo: $\square \operatorname{Sim} \square$ Não Ex-Etilismo: $\square \operatorname{Sim} \square$ Não

Período:_ Drogas ilícitas: $\square$ Sim $\square$ Não Tipo:__ Período:

Antecedentes cirúrgicos:

Antecedentes familiares:

Antecedentes pessoais: 


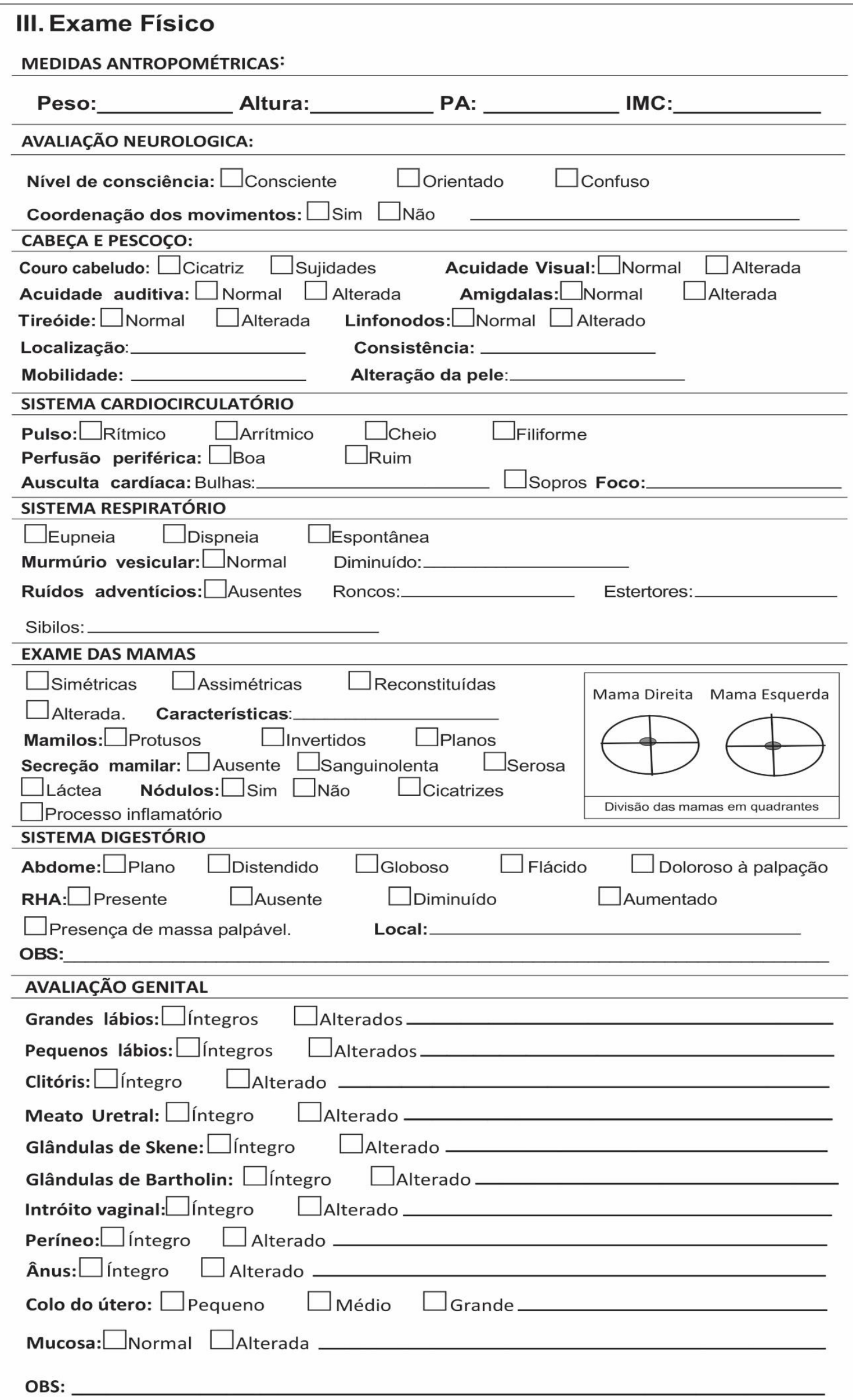




\section{POSSÍVEIS DIAGNÓSTICOS DE ENFERMAGEM: O ENFERMEIRO DEVE COMPLEMENTAR OS DIAGNÓSTICOS}

$\square$ Estilo de vida sedentário

$\square$ Manutenção ineficaz da saúde

$\square$ Risco de constipação

$\square$ Troca de gases prejudicada

$\square$ Padrão de sono prejudicado

$\square$ Mobilidade física prejudicada

$\square$ Padrão respiratório ineficaz

$\square$ Débito cardíaco diminuído

$\square$ Disposição para melhora do autocuidado

$\square$ Padrão de sexualidade ineficaz

$\square$ Risco de infecção

\section{INTERVENÇÕES}

